\title{
Evaluating the sensitivity and specificity of NEATstik technology compared to an activity-based immunoassay in sputum samples from participants with COPD
}

\author{
To the Editor:
}

Chronic bacterial infection may play an important role in the progression of COPD [1] with the pulmonary inflammatory response driven by neutrophils [2]. Neutrophil elastase (NE) is a serine protease stored and secreted by neutrophils [3] and is an essential defence against bacterial infection [4]. Proteases are produced and stored in the latent form and are only activated upon appropriate signalling [5]. Active sputum NE is a potential biomarker for infection in several respiratory diseases including, but not limited to, cystic fibrosis, COPD and bronchiectasis [6-10], with levels correlating to bacterial infection [11] and future risk of exacerbations in patients with bronchiectasis [12]. NE has been shown to be active in host defence against bacteria [4], both intracellularly and via extracellular traps [13]. NEATstik is a point of care test for NE which gives a qualitative result from sputum in $10 \mathrm{~min}$ with minimal processing. This may benefit clinical practice as a rapid indicator of bacterial infection and neutrophil activation.

We investigated the sensitivity of the NEATstik to correctly identify elevated NE levels (measured by the ProteaseTag active NE immunoassay) in participants with COPD at stable state. We also compared NE levels with symptoms, lung function and airway inflammation.

Participants entering an observational study completed lung function tests, health questionnaires using the COPD assessment test [14], symptoms using the (visual analogue score (VAS) [15] and, providing blood and spontaneous $(47 \%)$ or induced sputum samples (53\%) (research ethics committee reference 08/H0406/189). All samples $(\mathrm{n}=30)$ were collected at stable state. Sputum plugs were collected and processed immediately and a cell differential and bacterial load assessment (colony forming units) were obtained. An additional sputum plug was used to quantify active NE. The sputum plug was split to test with the NEATstik and ProteaseTag active NE immunoassay. All samples were processed as per manufacturer's instructions, which included a maximum sputum weight and volume of supernatant of $2 \mathrm{~g}$ and $10 \mathrm{~g}$ respectively. The median sputum weight used was $0.07 \mathrm{~g}$ (interquartile range (IQR) 0.03-0.164) for the NEATstik and $0.08 \mathrm{~g}(0.05-0.164)$ for the ProteaseTag active NE immunoassay. In brief, one portion of the sputum plug was diluted 10-fold in sample dilution buffer and $70 \mu \mathrm{L}$ of this solution was then removed and placed in the "sample port" on the NEATstik. The second portion of the sputum plug was diluted five-fold with PBS, mixed thoroughly and then centrifuged (at $4^{\circ} \mathrm{C}, 3000 \times g$ for $30 \mathrm{~min}$ ). The supernatant was removed and $\mathrm{NE}$ levels were measured using the ProteaseTag active NE immunoassay, following manufacturer's instructions. All samples were diluted 1 in 100 and run in duplicate. The standards were from $15.63 \mathrm{ng} \cdot \mathrm{mL}^{-1}$ to $1000 \mathrm{ng} \cdot \mathrm{mL}^{-1}$. An NE cut-off of $8000 \mathrm{ng} \cdot \mathrm{mL}^{-1}$, using the ProteaseTag assay was assigned positive as per the manufacturer's instruction. The positive predictive value and sensitivity of NEATstik to identify an elevated NE activity was analysed.

30 COPD participant samples were selected (males=20) with a mean age of 68 (range 45-82) years. The majority were ex-smokers $(\mathrm{n}=21)$ with a mean \pm sD smoking history and forced expiratory volume in $1 \mathrm{~s}\left(\mathrm{FEV}_{1}\right)$ of $47 \pm 45$ pack-years and $49 \pm 25 \%$ pred, respectively. A positive NEATstik was detected in five sputum samples. The median ProteaseTag NE was 820.8 (IQR 400.0-1354.0) ng. $\mathrm{mL}^{-1}$ and three samples had elevated values (above $8000 \mathrm{ng} \cdot \mathrm{mL}^{-1}$ ) and thus assigned positive; these were also positive using the NEATstik method. Table 1

@ERSpublications

NEATstik is a point of care test which gives a qualitative result on whether neutrophil elastase is elevated from sputum in $\mathbf{1 0}$ min with minimal processing and could be of benefit in clinical practice http://bit.ly/2SohUhf

Cite this article as: Thulborn SJ, Cane JL, Connolly C, et al. Evaluating the sensitivity and specificity of NEATstik technology compared to an activity-based immunoassay in sputum samples from participants with COPD. Eur Respir J 2020; 55: 1902412 [https://doi.org/10.1183/13993003.02412-2019]. 
TABLE 1 Comparison of lung function, participant health and inflammation across positive and negative groups utilising both the ProteaseTag and NeatStik tests

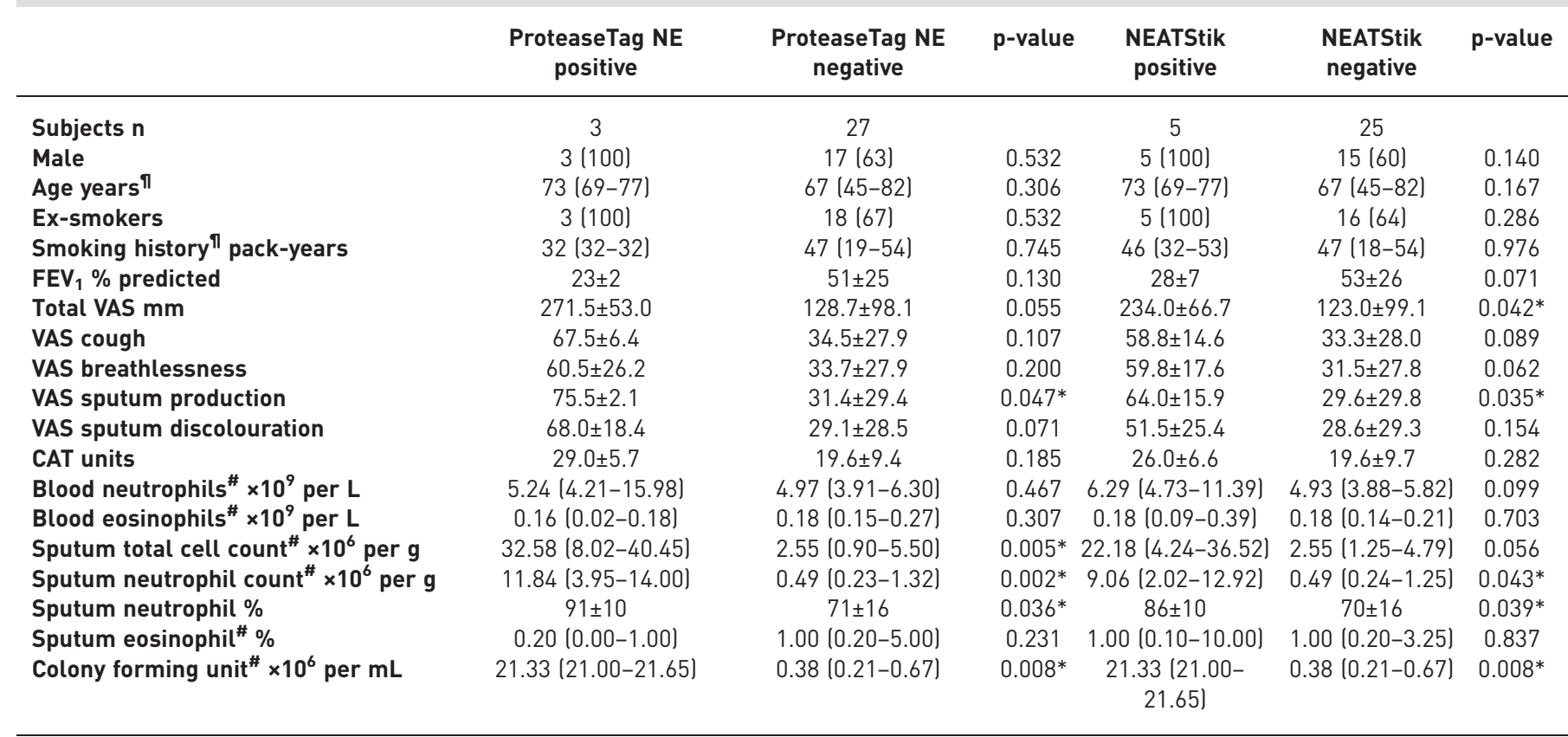

Data are presented as $\mathrm{n}(\%)$ or mean $\pm \mathrm{SD}$, unless otherwise stated. NE: neutrophil elastase; $\mathrm{FEV}_{1}$ : forced expiratory volume in $1 \mathrm{~s}$; VAS: visual analogue scale, performed on $100 \mathrm{~mm}$ line from "no symptoms" to "worst symptoms", higher scores represent worse symptoms (total score addition of measured domains: cough, dyspnoea, sputum production and sputum purulence); CAT: COPD assessment questionnaire, eight questions on a 1 to 5 point scale. ${ }^{\#}$ : median (interquartile range); ${ }^{\text {": }}$ mean (range). ${ }^{*}: p<0.05$.

shows the clinical and sputum characteristics of samples positive with NeatStik or ProteaseTag compared to those that were negative. Samples with elevated NE levels measured using both the NEATstik and ProteaseTag assay had significantly higher bacterial load and sputum neutrophils. The ProteaseTag assay also had a significantly higher sputum total cell count and the NEATStik had a higher total score on the VAS questionnaire. The individual VAS domains of cough, breathlessness, sputum production and sputum discolouration, demonstrated that symptoms of sputum production was significantly elevated in the samples with elevated NE levels. The sensitivity and specificity of NEATStik ${ }^{\oplus}$ to identify an elevated ProteaseTag ${ }^{\oplus} \mathrm{NE}$ was $100 \%$ (95\% CI 44-100) and 93\% (95\% CI 77-99), respectively, with a positive and negative predictive value of $60 \%$ and $100 \%$, respectively. ProteaseTag NE correlated with airway inflammation (sputum total cell count $\mathrm{r}=0.390, \mathrm{p}=0.048$ and absolute sputum neutrophils $\mathrm{r}=0.435, \mathrm{p}=0.026)$ and bacterial load $(\mathrm{r}=0.650$, $\mathrm{p}=0.002$ ), with a trend to high ProteaseTag NE correlating with worsened lung function $\left(\mathrm{FEV}_{1} \%\right.$ predicted $\mathrm{r}=$ $-0.264, \mathrm{p}=0.203$ ) and VAS symptoms (VAS $\mathrm{r}=0.372, \mathrm{p}=0.067$ ). There was no significant difference between $\mathrm{NE}$ levels in ex- or current smokers ( $\mathrm{p}=0.555)$.

NEATstik is sensitive and specific for identifying an elevated active NE level, and this was linked to increased sputum neutrophil counts, bacterial load in the lungs and symptoms of sputum production. The NEATstik has potential to be a rapid method to identify bacterial infection. The NE ProteaseTag cut-off used was first identified in patients with bronchiectasis patients [7] and thus may underestimate infection in patients with COPD. Additional analysis at lower cut-offs may thus be required.

Samantha J. Thulborn ${ }^{1,2}$, Jennifer L. Cane ${ }^{1,2}$, Clare Connolly ${ }^{1,2}$, Catherine Borg ${ }^{1,2}$, Kelly L. Moffitt ${ }^{3}$, David Ribeiro ${ }^{3}$, Charlene Robb ${ }^{3}$, Richard E.K. Russell $\oplus^{1}$ and Mona Bafadhel ${ }^{1}$

${ }^{1}$ Respiratory Medicine Unit, Nuffield Dept of Medicine, University of Oxford, Oxford, UK. ${ }^{2}$ Oxford NIHR Biomedical Research Centre, University of Oxford, Oxford, UK. ${ }^{3}$ ProAxsis Ltd, Belfast, UK.

Correspondence: Mona Bafadhel, University of Oxford, Dept of Respiratory Medicine, Nuffield Dept of Medicine, Old Road Campus, Oxford, OX3 7FZ, UK. E-mail: mona.bafadhel@ndm.ox.ac.uk

Received: 16 Dec 2019 | Accepted after revision: 30 Jan 2020

Conflict of interest: S.J. Thulborn has nothing to disclose. J.L. Cane has nothing to disclose. C. Connolly has nothing to disclose. C. Borg has nothing to disclose. K.L. Moffitt has nothing to disclose. D. Ribeiro is an employee of ProAxsis 
Limited, which manufactures both the NEATstik test and the ProteaseTag Active Neutrophil Elastase immunoassay. C. Robb has nothing to disclose. R.E.K. Russell reports personal fees from Boehringer Ingelheim Ltd, Chiesi Pharma Ltd, GlaxoSmithKline Ltd and Teva Uk Ltd, grants from Circassia Ltd, grants and personal fees from AstraZeneca Ltd, outside the submitted work. M. Bafadhel reports grants from NIHR, during the conduct of the study; personal fees and non-financial support for advisory board work, educational symposia and travel to conferences from AZ, BI, Chiesi, GSK, Novartis and Teva, outside the submitted work.

Support statement: This work was funded by the Medical Research Council, National Institute for Health Research (Respiratory Theme), Oxfordshire Health Services Research Committee, Wellcome Trust and Academy of Medical Sciences. Funding information for this article has been deposited with the Crossref Funder Registry.

\section{References}

1 Sethi S, Murphy TF. Bacterial infection in chronic obstructive pulmonary disease in 2000: a state-of-the-art review. Clin Microbiol Rev 2001; 14: 336-363.

2 Bafadhel M, Haldar K, Barker B, et al. Airway bacteria measured by quantitative polymerase chain reaction and culture in patients with stable COPD: relationship with neutrophilic airway inflammation, exacerbation frequency, and lung function. Int J Chron Obstruct Pulmon Dis 2015; 10: 1075-1083.

3 Döring G. The role of neutrophil elastase in chronic inflammation. Am J Respir Crit Care Med 1994; 150: S114-S117.

$4 \quad$ Drescher B, Bai F. Neutrophil in viral infections, friend or foe? Virus Res 2013; 171: 1-7.

5 Chua F, Laurent GJ. Neutrophil elastase: mediator of extracellular matrix destruction and accumulation. Proc Am Thorac Soc 2006; 3: 424-427.

6 Brusselle GG, Braeckel EV. Sputum neutrophil elastase as a biomarker for disease activity in bronchiectasis. Am J Respir Crit Care Med 2017; 195: 1289-1291.

7 Chalmers JD, Moffitt KL, Suarez-Cuartin G, et al. Neutrophil elastase activity is associated with exacerbations and lung function decline in bronchiectasis. Am J Respir Crit Care Med 2017; 195: 1384-1393.

8 Crocetti L, Quinn M, Schepetkin I, et al. A patenting perspective on human neutrophil elastase (HNE) inhibitors (2014-2018) and their therapeutic applications. Expert Opin Ther Pat 2019; 29: 555-578.

9 Muhlebach MS, Clancy JP, Heltshe SL, et al. Biomarkers for cystic fibrosis drug development. J Cyst Fibros 2016; 15: 714-723.

10 Thulborn SJ, Mistry V, Brightling CE, et al. Neutrophil elastase as a biomarker for bacterial infection in COPD. Respir Res 2019; 20: 170.

11 Keir HR, Fong CJ, Dicker AJ, et al. Profile of the ProAxsis active neutrophil elastase immunoassay for precision medicine in chronic respiratory disease. Expert Rev Mol Diagn 2017; 17: 875-884.

12 Shoemark A, Cant E, Carreto L, et al. A point-of-care neutrophil elastase activity assay identifies bronchiectasis severity, airway infection and risk of exacerbation. Eur Respir J 2019; 53: 1900303.

13 Pham CTN. Neutrophil serine proteases: specific regulators of inflammation. Nat Rev Immunol 2006; 6: 541-550.

14 Gupta N, Pinto LM, Morogan A, et al. The COPD assessment test: a systematic review. Eur Respir J 2014; 44: 873-884.

15 Brightling CE, Monterio W, Green RH, et al. Induced sputum and other outcome measures in chronic obstructive pulmonary disease: safety and repeatability. Respir Med 2001; 95: 999-1002. 